\title{
The Innate Immune Receptor CD14 Mediates Lymphocyte Migration in EAE
}

\author{
Ramona Halmer ${ }^{\mathrm{a}}$ Laura Davies ${ }^{\mathrm{b}}$ Yang Liu ${ }^{\mathrm{a}}$ Klaus Fassbender ${ }^{\mathrm{a}}$ Silke Walter \\ aDepartment of Neurology, Saarland University Hospital, Homburg, Germany; 'Department of \\ Neuropathology, University of Calgary, Calgary, Canada
}

\section{Key Words}

Multiple sclerosis - Experimental autoimmune encephalomyelitis - LPS receptor T cell activation - Blood brain barrier

\begin{abstract}
Background: Multiple sclerosis is the most common autoimmune disease of the central nervous system in young adults and histopathologically characterized by inflammation, demyelination and gliosis. It is considered as a $\mathrm{CD}^{+} \mathrm{T}$ cell-mediated disease, but also a disease-promoting role of the innate immune system has been proposed, based e.g. on the observation that innate immune receptors modulate disease severity of experimental autoimmune encephalomyelitis. Recent studies of our group provided first evidence for a key role of the innate immune LPS receptor (CD14) in pathophysiology of experimental autoimmune encephalomyelitis. CD14-deficient experimental autoimmune encephalomyelitis mice showed increased clinical symptoms and enhanced infiltration of monocytes and neutrophils in brain and spinal cord. Methods: In the current study, we further investigated the causes of the disease aggravation by CD14-deficiency and examined T cell activation, also focusing on the costimulatory molecules CTLA-4 and CD28, and T cell migration capacity over the blood brain barrier by FACS analysis, in vitro adhesion and transmigration assays. Results: In the results, we observed a significantly increased migration of CD14-deficient lymphocytes across an endothelial monolayer. In contrast, we did not see any differences in expression levels of TCR/CTLA-4 or TCR/CD28 and lymphocyte adhesion to endothelial cells from CD14-deficient compared to wildtype mice. Conclusion: The results demonstrate an important role of CD14 in migration of lymphocytes, and strengthen the importance of innate immune receptors in adaptive immune disorders, such as multiple sclerosis.




\section{Cellular Physiology Cell Physiol Biochem 2015;37:269-275

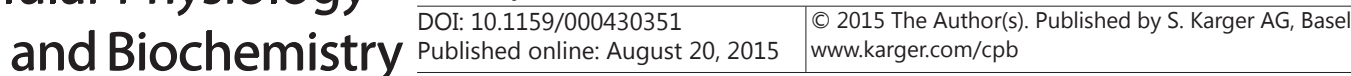 \\ Halmer/Davies/Liu/Fassbender/Walter: CD14 in EAE}

\section{Introduction}

Multiple sclerosis (MS) is a chronic autoimmune disease of the central nervous system (CNS), characterized by inflammation, demyelination and axonal degeneration [1]. Most of the pathophysiological understanding has been gained by its animal model experimental autoimmune encephalomyelitis (EAE).

As disease-initiating step, T cells are activated by presentation of myelin components by antigen-presenting cells to the T cell receptor (TCR) [2]. Additional co-stimulatory signals via B7/CD28/CTLA-4 pathways are required. Thereby, activation via CD28 leads to proinflammatory response [3], while T cell activation via CTLA-4, leads to anti-inflammatory signals [4]. Migration of the activated T cells across the blood-brain barrier (BBB) is crucial in disease development and follows a complex multi-step cascade [5, 6]. Recent studies showed a protective role of the innate immune receptor CD14 in murine Streptococcus pneumoniae meningitis by lowered migration capacity of leukocytes [7].

Although MS is considered as $\mathrm{CD}^{+} \mathrm{T}$ cell-mediated disease, several findings support a pathophysiological role of the innate immune system. Recently, we described that CD14deficiency resulted in increased disease severity and inflammatory infiltration in EAE. These results suggest that $\mathrm{CD} 14$ has protective effects in this autoimmune disease [8]. Up to now, the pathophysiological mechanism remains unclear. Here, we investigate the pathophysiological function of CD14 in T cell activation and migration across the inflamed BBB.

\section{Material and Methods}

Mice

Female CD14-deficient mice and wildtype (wt) littermates or C57BL/6J mice were kept in our breeding facility, purchased from Jackson Laboratories via Charles River Laboratories (Sulzfeld, Germany). All animal procedures were performed in compliance with the German Guide for the Care and Use of Laboratory Animals.

Induction of active EAE

6-8 week-old mice were subcutaneously immunized with $300 \mu \mathrm{g}$ myelin oligodendrocyte glycoprotein $_{\text {aa35-55 }}$ (Charité Medical University, Berlin, Germany) in complete Freund's adjuvant, followed by boostering with 300 ng Bordetella pertussis toxin (Enzo Life Sciences GmbH, Lörrach, Germany) intraperitoneally on days 0 and 2. Daily body weights and clinical disease scores were assessed: $0=$ healthy, $0.5=\operatorname{limp}$ tail, 1 = hindleg weakness, $2=$ hindleg paraparesis, $3=$ hindleg paraparesis and incontinence, 4 = tetraparesis, 5 = death. Mice were sacrificed at day 10 (preclinical phase) and peak of disease (clinical phase).

Lymphocyte adhesion assay

16-well-chamberslides (VWR GmbH, Darmstadt, Germany) were coated with $50 \mu \mathrm{g} / \mathrm{ml}$ fibronectin (Roche Diagnostics GmbH, Mannheim, Germany) and plated with 2x104 mouse endothelial cells bEnd.3/

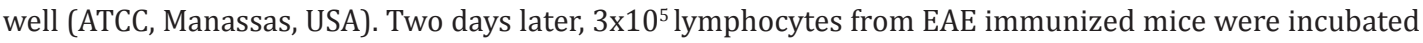
on this monolayer while rocking $\left(40\right.$ minutes, $4^{\circ} \mathrm{C}$ ). Assays were washed and fixed in $2.5 \%$ glutaraldehyde $\left(2\right.$ hours, $4^{\circ} \mathrm{C}$ ). Assays were analyzed by counting the adherent cells by microscope. Values of adherent wt lymphocytes of each phase were defined as $100 \%$.

\section{Lymphocyte migration assay}

Inserts of transwells with $5 \mu \mathrm{m}$ pore size (VWR GmbH) were coated with $50 \mu \mathrm{g} / \mathrm{ml}$ Laminin and seeded with bEnd. 3 cells two days prior to the assay. $1 \times 10^{6}$ EAE lymphocytes were added to the inserts. Assays were incubated for 4 hours. Migrated cells in the lower compartment were analyzed by TruCount tubes and flow cytometry (FACS Canto II, BD Biosciences, Heidelberg, Germany) according to the manufacturer's instructions (BD Biosciences). Inserts were stained with Giemsa for bEnd.3 monolayers. Values of migrated wt lymphocytes of each phase were defined as $100 \%$. 


\section{Cellular Physiology Cell Physiol Biochem 2015;37:269-275

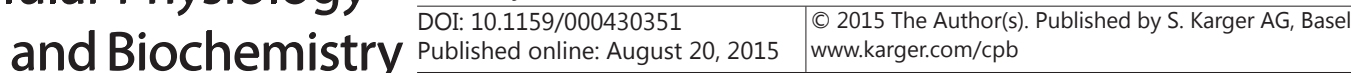 \\ Halmer/Davies/Liu/Fassbender/Walter: CD14 in EAE}

\section{Flow cytometry}

EAE lymphocytes were stained with anti-CD4 FITC, anti-CD8 PerCP Cy5.5, anti-TCR $\beta$ PE, anti-CTLA-4 purified, anti- $\alpha 4$-integrin biotin, anti-LFA-1 $\alpha$ chain PE labelled (all from BD Biosciences) and anti-CD28 APC (BIOZOL Diagnostica GmbH, Eching, Germany). Purified anti-CTLA-4 was followed by incubation with Cy5ß-conjugated goat anti-armenian hamster IgG (Jackson ImmunoResearch Europe Ltd., Suffolk, UK). Biotinylated anti- $\alpha 4$-integrin was detected with APC-conjugated Streptavidin (BD Biosciences). Samples were analyzed by flow cytometry with FACS Diva Software.

\section{Statistical analysis}

Data are expressed as means \pm SD. Statistical analysis was performed with ANOVA testing. * $p \leq 0.05$ has been considered as statistical significant result.

\section{Results}

\section{CD14-deficiency increases transmigration of EAE lymphocytes in vitro}

Based on the knowledge that CD14-deficient mice show a modified clinical disease course [8] compared to wt mice, we analyzed transmigration capability of EAE derived lymphocytes from CD14-deficient and wt mice in vitro. EAE lymphocytes contained $18.8 \%$ $\mathrm{CD}^{+}$and $18.1 \% \mathrm{CD}^{+} \mathrm{T}$ cells as determined by FACS analysis (data not shown). We observed a significantly increased migration capability of lymphocytes from EAE immunized CD14deficient compared to wt mice, when lymphocytes were isolated in the clinical phase of the disease.

Clinical phase was defined as the day of disease course when CD14-deficient mice showed maximal clinical symptoms (wt: score $0.73 \pm 0.15$; CD14-/-: score $1.70 \pm 0.12$; day $18.60 \pm 0.59$ ).

Lymphocytes derived from non-immunized control animals or mice during preclinical phase did not show any baseline difference (wt migration capacity was defined as $100 \%$ for each phase; Fig. 1).

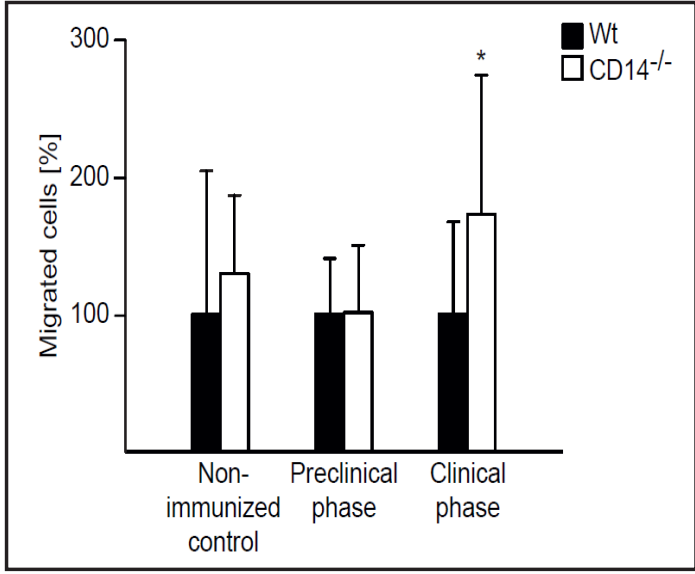

Fig. 1. Migration capability of lymphocytes derived from EAE immunized CD14-deficient mice $(n=15)$ is significantly increased in the clinical phase of the disease as compared to wildtype (wt) mice ( $\mathrm{n}=13$; $\mathrm{p}=0.0155$ ). In contrast, there was no difference in non-immunized controls (wt $n=5 ; \operatorname{CD} 14 \% \mathrm{n}=5$ ) or during preclinical phase (wt $n=7$; CD14\% $n=7$ ). Values of migrated wt lymphocytes of each phase were defined as $100 \%$. Shown are means \pm SD. ${ }^{*} \mathrm{p} \leq 0.05$.

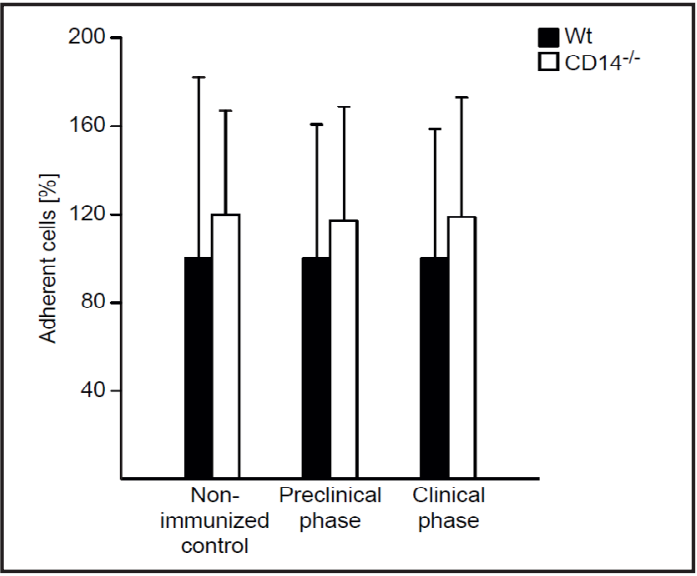

Fig. 2. In vitro adhesion assays showed no difference in adhesion capacity to bEnd. 3 cells between lymphocytes from CD14-deficient and wildtype (wt) mice in control (wt $n=6-10 ;$ CD14\% $n=6-11$ ). Values of adherent wt lymphocytes of each phase were defined as $100 \%$. Shown are means \pm SD. 
Fig. 3. We detected no significant difference in (a) TCR/ CTLA-4 expression on $\mathrm{CD}^{+}$ and $\mathrm{CD} 8^{+}$lymphocytes from CD14-deficient compared to wildtype (wt) mice (wt $n=3$ 8; $\mathrm{CD} 14^{-1} \mathrm{n}=3-$ 6). CTLA-4 expression was lowered in the clinical phase. CD14-deficiency does not affect expression levels of (b) TCR/CD28 on $\mathrm{CD}^{+}$and $\mathrm{CD}^{+}$ cells compared to wt mice (wt $\mathrm{n}=4-5$; $\quad$ CD14 $\%$ $n=4-5$ ). Shown are means \pm SD.

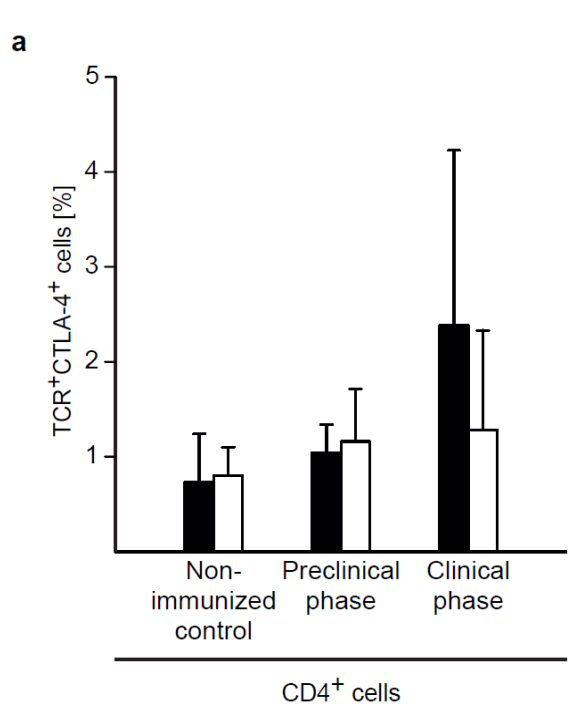

b

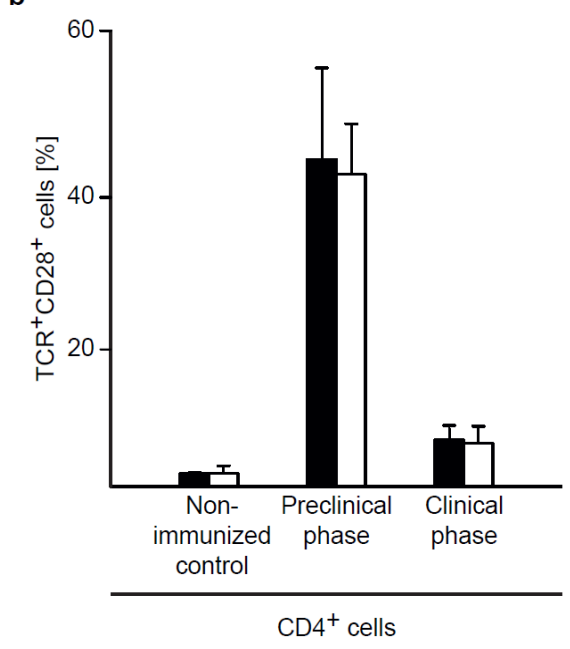

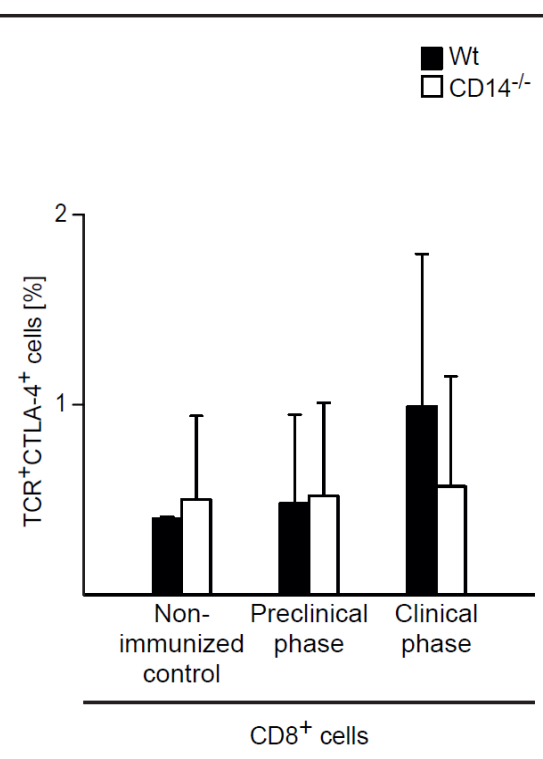

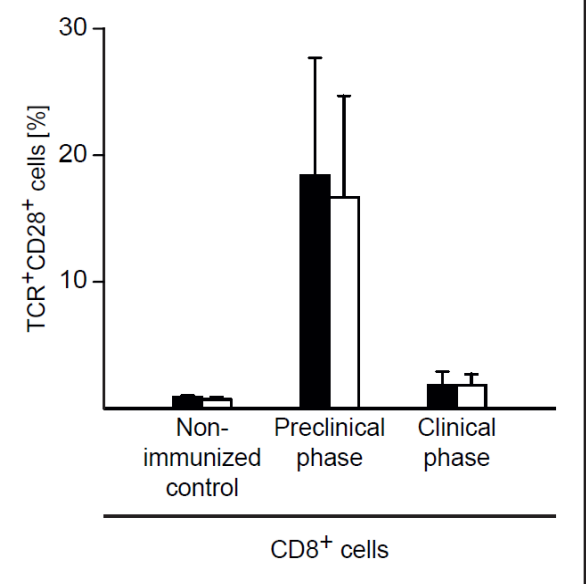

CD14-deficiency does not increase lymphocyte adhesion capacity in vitro

Adhesion capacity of lymphocytes from CD14-deficient compared to wt EAE mice showed neither any difference in preclinical nor clinical phase as detected by in vitro adhesion assays (wt adhesion defined as 100\%; Fig. 2).

CD14-deficiency does not influence lymphocyte activation or adhesion molecule expression

CD14-deficient $\mathrm{CD} 4^{+}$and $\mathrm{CD} 8^{+} \mathrm{T}$ cells did not show any difference in the expression levels of TCR/CTLA-4 or TCR/CD28 compared to wt cells (Fig. 3). Interestingly, expression of TCR/CD28 was significantly lower in the clinical phase compared to the preclinical phase independent from genetic background and cell type (Fig. 3). T cells derived from nonimmunized CD14-deficient and wt mice did not show any difference in baseline levels (Fig.3).

Leukocyte function-associated antigen (LFA-1) and $\alpha 4 \beta 1$-integrin are necessary for firm adhesion during the multi-step process [9]. We detected neither a difference of LFA$1 \alpha$ chain nor $\alpha 4$ expression on lymphocytes from CD14-deficient mice compared to wt EAE lymphocytes during preclinical or clinical phase (Fig. 4). LFA-1 expression was constantly at high levels independent from EAE immunization, while $\alpha 4$ levels depended on disease phases (Fig. 4). 
Fig. 4. We detected neither a difference of the adhesion molecules (a) $\alpha 4$ expression nor (b) LFA- $1 \alpha$ chain on lymphocytes from CD14-deficient compared to wildtype (wt) lymphocytes in controls (wt $n=4$ 8; CD14\% $\mathrm{n}=4-6$ ). LFA-1 expression was constantly at high levels independent from EAE immunization. $\alpha 4$ levels increased in the preclinical phase after immunization and decreased significantly in the clinical phase after development of EAE symptoms. Shown are means \pm SD.
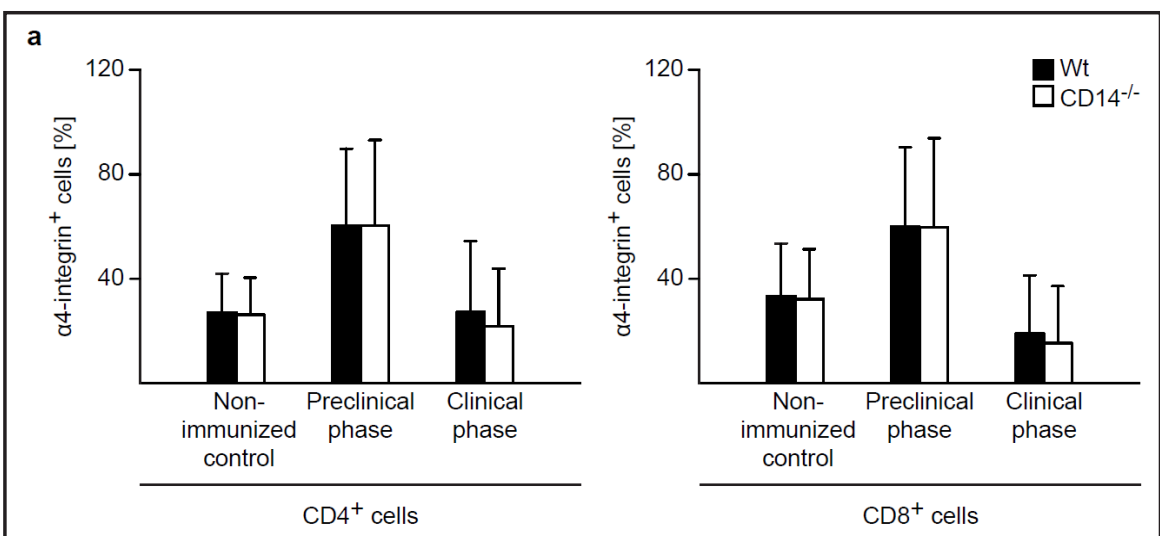

b

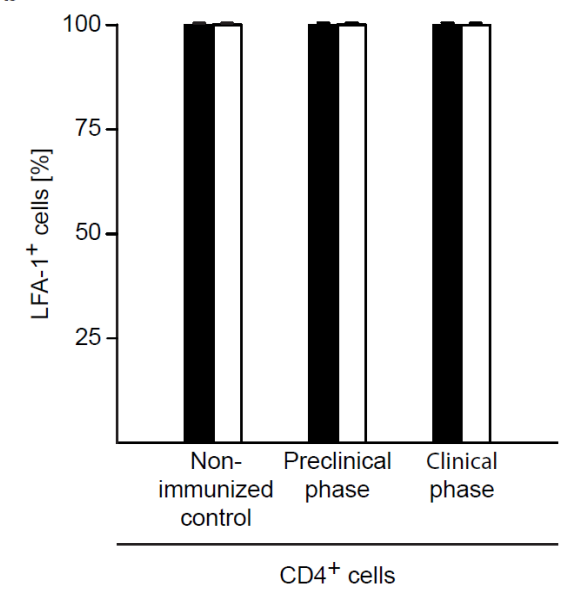

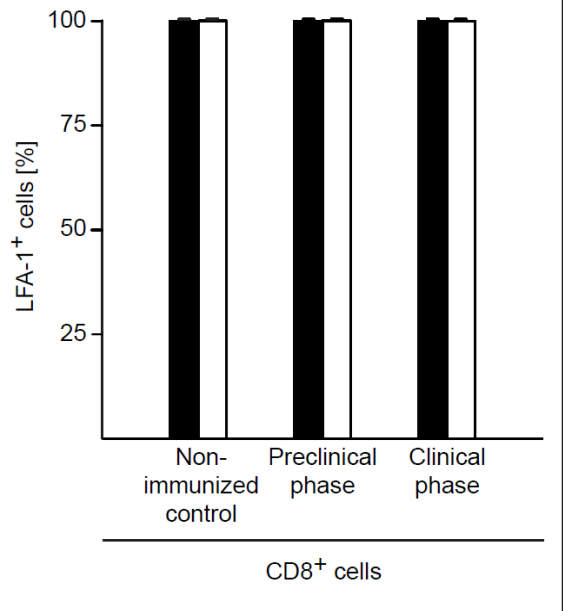

\section{Discussion}

Leukocyte adhesion to and migration through the BBB are crucial steps in the pathophysiology of MS and EAE [10]. The results of this study demonstrate a significantly increased $\mathrm{T}$ cell migration capacity of CD14-deficient lymphocytes through an endothelial cell layer in vitro. This is a possible explanation for the observed increase in clinical and histopathological features of EAE in CD14-deficient animals [8]. Our findings are similar to earlier descriptions, showing an enhanced transmigration of leukocytes from CD14-deficient mice across the BBB in pneumococcal meningitis [7].

In line with earlier descriptions [11], we did not observe any difference in adhesion capacity in our in vitro system, which focused on the interaction between VLA- 4 and VCAM-1 as assays were performed at $4^{\circ} \mathrm{C}$, thereby preventing LFA- 1 operation [12]. Involvement of LFA-1/ICAM-1 as possible explanation for the in vitro observation needs further investigation and is one limitation of our work. Beside, we excluded any influence of CD14 on adhesion molecule expression of LFA- $1 \alpha$ chain and $\alpha 4$. LFA- 1 expression was maximal throughout EAE disease course and even in mice without immunization. This is in contrast to earlier descriptions, demonstrating an increase of LFA-1 with immunization and ongoing disease [13]. Interestingly, mainly Th17 cell immigration into the CNS has been described to be dependent on LFA-1 [14]. Additionally, we detected increased levels of $\alpha 4$ in the pre-clinical phase after immunization. Independent from the genetic phenotype $\alpha 4$ levels decreased significantly in the clinical phase after development of EAE symptoms compared to the preclinical phase at day 10.

In our study, analysis of the co-stimulatory signals CTLA-4 and CD28 revealed no difference in the expression level between CD14-deficient and wt littermate mice. We saw 


\section{Cellular Physiology Cell Physiol Biochem 2015;37:269-275 \begin{tabular}{ll|l} 
DOI: 10.1159/000430351 20,2015 & $\begin{array}{l}\text { O) } 2015 \text { The Author(s). Published by S. Karger AG, Basel } \\
\text { www.karger.com/cpb }\end{array}$ \\
\hline and Biochemistry & Published online: August 20,
\end{tabular} \\ Halmer/Davies/Liu/Fassbender/Walter: CD14 in EAE}

a significant reduction of CD28 expression in lymph nodes in both genetic phenotypes at the clinical peak compared to the preclinical phase of EAE development. This together with reduced $\alpha 4$ levels might be caused by migration of inflammatory reactive T cells into the CNS rather than persisting in peripheral lymph nodes. This is strengthened by the finding that CD28 is elevated in the CNS at the peak of EAE [15]. Our study excluded a significant difference in the expression of CTLA- 4 on both $\mathrm{CD}^{+}$and $\mathrm{CD} 8^{+} \mathrm{T}$ cells derived from CD14-deficient or wt littermates. Nevertheless, CTLA-4 expression was lowered in the clinical phase, indicating a reduced anti-inflammatory potential [16]. Taken together, the results exclude an altered expression of adhesion molecules or $\mathrm{T}$ cell activation molecules as a reason for enhanced migration of CD14-deficient lymphocytes.

Another possible explanation for the increased disease activity and more pronounced EAE typical features in CD14-deficient animals is an altered concentration of chemokines leading to enhanced migration across the inflamed BBB. Interestingly, an earlier study postulated increased levels of the macrophage inflammatory protein-2 (MIP-2) in brain homogenates of CD14-deficient mice explaining the enhanced migration in pneumococcal meningitis [7]. The authors also showed released expression of the CXC chemokine receptor 2 (CXCR2) and observed that blocking of CXCR2 abolished earlier death of CD14-deficient mice. In this context, they discussed that CD14 may control migration by regulating TLR2 mediated CXCR2 expression [7]. As a glycosylphosphatidyl-inositol-anchored protein without transmembrane and intracellular domains CD14 cannot transmit signals on its own and interacts with Toll-like receptors for signal transduction [17]. Concerning EAE, the pathophysiological mechanism of increased migration of CD14-deficient lymphocytes is up to now unknown and remains to be further investigated.

\section{Conclusion}

Taken together, our findings demonstrate an important role of CD14 in migration of lymphocytes. This might be one possible explanation for the earlier described disease course modification and increase in inflammatory infiltrates in CD14-deficient mice. The clinical value of these findings needs to be further investigated.

\section{Disclosure Statement}

The authors declare that they have nothing to disclose.

\section{References}

1 Trapp BD, Peterson J, Ransohoff RM, Rudick R, Mork S, Bo L: Axonal transection in the lesions of multiple sclerosis. N Engl J Med 1998;338:278-285.

2 Meuer SC, Acuto O, Hercend T, Schlossman SF, Reinherz EL: The human t-cell receptor. Annu Rev Immunol 1984;2:23-50.

3 Viola A, Schroeder S, Sakakibara Y, Lanzavecchia A: T lymphocyte costimulation mediated by reorganization of membrane microdomains. Science 1999;283:680-682.

4 Linsley PS, Greene JL, Tan P, Bradshaw J, Ledbetter JA, Anasetti C, Damle NK: Coexpression and functional cooperation of ctla-4 and cd28 on activated t lymphocytes. J Exp Med 1992;176:1595-1604.

5 Engelhardt B, Ransohoff RM: The ins and outs of t-lymphocyte trafficking to the cns: Anatomical sites and molecular mechanisms. Trends Immunol 2005;26:485-495.

6 Ley K, Laudanna C, Cybulsky MI, Nourshargh S: Getting to the site of inflammation: The leukocyte adhesion cascade updated. Nat Rev Immunol 2007;7:678-689. 


\section{Cellular Physiology Cell Physiol Biochem 2015;37:269-275 \begin{tabular}{l|l} 
DOI: 10.1159/000430351 20, 2015 & $\begin{array}{l}\text { O 2015 The Author(s). Published by S. Karger AG, Basel } \\
\text { www.karger.com/cpb }\end{array}$ \\
and Biochemistry Published online: August 20.
\end{tabular} \\ Halmer/Davies/Liu/Fassbender/Walter: CD14 in EAE}

7 Echchannaoui H, Frei K, Letiembre M, Strieter RM, Adachi Y, Landmann R: Cd14 deficiency leads to increased mip-2 production, cxcr2 expression, neutrophil transmigration, and early death in pneumococcal infection. J Leukoc Biol 2005;78:705-715.

8 Walter S, Doering A, Letiembre M, Liu Y, Hao W, Diem R, Bernreuther C, Glatzel M, Engelhardt B, Fassbender $\mathrm{K}$ : The lps receptor, cd14, in experimental autoimmune encephalomyelitis and multiple sclerosis. Cell Physiol Biochem 2006;17:167-172.

9 Engelhardt B, Ransohoff RM: Capture, crawl, cross: The t cell code to breach the blood-brain barriers. Trends Immunol 2012;33:579-589.

10 Engelhardt B: The blood-central nervous system barriers actively control immune cell entry into the central nervous system. Curr Pharm Des 2008;14:1555-1565.

11 Kerfoot SM, Long EM, Hickey MJ, Andonegui G, Lapointe BM, Zanardo RC, Bonder C, James WG, Robbins SM, Kubes P: Tlr4 contributes to disease-inducing mechanisms resulting in central nervous system autoimmune disease. J Immunol 2004;173:7070-7077.

12 Wysocki J, Issekutz TB: Effect of t cell activation on lymphocyte-endothelial cell adherence and the role of vla-4 in the rat. Cell Immunol 1992;140:420-431.

13 Selmaj K, Walczak A, Mycko M, Berkowicz T, Kohno T, Raine CS: Suppression of experimental autoimmune encephalomyelitis with a tnf binding protein (tnfbp) correlates with down-regulation of vcam-1/vla-4. Eur J Immunol 1998;28:2035-2044.

14 Rothhammer V, Heink S, Petermann F, Srivastava R, Claussen MC, Hemmer B, Korn T: Th17 lymphocytes traffic to the central nervous system independently of alpha4 integrin expression during eae. J Exp Med 2011;208:2465-2476.

15 Issazadeh S, Navikas V, Schaub M, Sayegh M, Khoury S: Kinetics of expression of costimulatory molecules and their ligands in murine relapsing experimental autoimmune encephalomyelitis in vivo. J Immunol 1998;161:1104-1112.

16 Karandikar NJ, Vanderlugt CL, Walunas TL, Miller SD, Bluestone JA: Ctla-4: A negative regulator of autoimmune disease. J Exp Med 1996;184:783-788.

17 Pugin J, Heumann ID, Tomasz A, Kravchenko VV, Akamatsu Y, Nishijima M, Glauser MP, Tobias PS, Ulevitch RJ: Cd14 is a pattern recognition receptor. Immunity 1994;1:509-516. 